This is an electronic reprint of the original article. This reprint may differ from the original in pagination and typographic detail.

Author(s): Kotimäki, Ville; Räsänen, Esa; Hennig, Holger; Heller, Eric J

Title: $\quad$ Fractal dynamics in chaotic quantum transport

Year: $\quad 2013$

Version:

Please cite the original version:

Kotimäki, V., Räsänen, E., Hennig, H., \& Heller, E. J. (2013). Fractal dynamics in chaotic quantum transport. Physical Review E, 88(2), Article 022913. https://doi.org/10.1103/PhysRevE.88.022913

All material supplied via JYX is protected by copyright and other intellectual property rights, and duplication or sale of all or part of any of the repository collections is not permitted, except that material may be duplicated by you for your research use or educational purposes in electronic or print form. You must obtain permission for any other use. Electronic or print copies may not be offered, whether for sale or otherwise to anyone who is not an authorised user. 


\title{
Fractal dynamics in chaotic quantum transport
}

\author{
V. Kotimäki, ${ }^{1}$ E. Räsänen, ${ }^{1,2,3,{ }^{*}}$ H. Hennig, ${ }^{3}$ and E. J. Heller ${ }^{3,4}$ \\ ${ }^{1}$ Nanoscience Center, Department of Physics, University of Jyväskylä, FI-40014 Jyväskylä, Finland \\ ${ }^{2}$ Department of Physics, Tampere University of Technology, FI-33101 Tampere, Finland \\ ${ }^{3}$ Department of Physics, Harvard University, Cambridge, Massachusetts 02138, USA \\ ${ }^{4}$ Department of Chemistry and Chemical Biology, Harvard University, Cambridge, Massachusetts 02138, USA
}

(Received 21 January 2013; published 13 August 2013)

\begin{abstract}
Despite several experiments on chaotic quantum transport in two-dimensional systems such as semiconductor quantum dots, corresponding quantum simulations within a real-space model have been out of reach so far. Here we carry out quantum transport calculations in real space and real time for a two-dimensional stadium cavity that shows chaotic dynamics. By applying a large set of magnetic fields we obtain a complete picture of magnetoconductance that indicates fractal scaling. In the calculations of the fractality we use detrended fluctuation analysis - a widely used method in time-series analysis - and show its usefulness in the interpretation of the conductance curves. Comparison with a standard method to extract the fractal dimension leads to consistent results that in turn qualitatively agree with the previous experimental data.
\end{abstract}

DOI: 10.1103/PhysRevE.88.022913

PACS number(s): 05.45.Df, 05.45.Pq, 73.23.Ad, 73.63.Kv

\section{INTRODUCTION}

Since the pioneering works of Mandelbrot [1], fractal patterns have been found in a variety of objects in nature including, e.g., snowflakes, fern leaves, coastlines [2,3], and even music [4-7]. These self-similar (or self-affine) structures were also found in many branches of chemistry and physics; prominent examples are crystal growth and fractal surfaces, and transport in gold nanowires and electron "billiards" $[3,8-15]$. In contrast with idealized mathematical fractals continuing to infinitely small scales, fractal scaling in nature has a lower and an upper limit.

While fractals found in nature are often well described by classical theories $[1,3,8,9]$, fractals have also been suggested to manifest in different quantum systems [16-21], where a fundamental lower cutoff for fractal scaling is given by the Heisenberg uncertainty principle. In the case of transport through chaotic systems, such as chaotic electron billiards, both semiclassical [22] (involving quantum interference) and classical mechanisms [23] for the emergence of fractal scaling have been proposed.

For quantum systems with an underlying classically mixed phase space with both regular and chaotic regions, a quantum graph model suggests a splitting of the chaotic regime into two parts [18]: One part yields fractal conductance fluctuations while the other one leads to isolated resonances on small scales. These isolated resonances were later shown to be associated with the eigenstates of a closed system [24].

A stadium quantum billiard of charged particles is a generic chaotic system whose underlying classical phase space is chaotic. The phase space becomes mixed in the presence of a (perpendicular) magnetic field. In the past two decades the system has been subject to several experiments [10,25-28]. A typical setup consists of the two-dimensional (2D) electron gas (2DEG) in a semiconductor heterostructure, where metallic gates are used to form the geometrical shape of the billiards, here called a quantum dot. Alternatively, stadium billiards (and

*esa.rasanen@tut.fi other chaotic systems) can be realized experimentally with microwave cavities [28].

The dynamics in chaotic cavities has been extensively studied with various theoretical methods including, e.g., random matrix theory [29], trajectory-based semiclassical theory [30], quantum mechanical kicked-rotor models [31], and tight-binding calculations [21]. Semiclassical and random matrix theory have been used to investigate weak localization and Ehrenfest time effects [32,33], while the kicked-rotor model and tight-binding studies have focused on the fractal structure of the quantum survival probability in chaotic cavities and the effect of changing the width of the output leads [21], respectively. Benenti et al. provide evidence for fractal fluctuations of the quantum survival probability in the nonclassical situation of strong localization [19]. However, previous dynamical approaches have not focused on conductance calculations in $2 \mathrm{D}$ chaotic cavities described by real-space grids in space and time.

It is worthwhile to notice that, in principle, the conductance problem of a chaotic cavity can be treated within the conventional transport formalism, where the equilibrium current is obtained time independently [34]. In this approach, the coupling matrix of the cavity eigenstates and the lead states need to be evaluated. The most tedious part is an accurate and efficient treatment of the 2D eigenvalue problem for the chaotic cavity in real space and in the presence of the magnetic field. Recent progress has been made in this direction [35] and such a conventional transport scheme is a subject for future work. Nevertheless, as shown below, the present dynamical approach provides an efficient way to assess the conductivity and gives also additional information on time-dependent effects in the system.

In this work we calculate the fractal scaling of conductance fluctuations in an open quantum stadium billiard in a full 2D model in real space and real time. Our explicit solution of the time-dependent Schrödinger equation for chaotic transport goes beyond both the semiclassical treatment [22] and the above mentioned quantum graph model [18]. We analyze the fractal scaling using two methods that originate from different fields of physics: the variation method $[3,10,36]$ and 
detrended fluctuation analysis [37-39] (DFA). The variation method was used by Sachrajda et al. [10] for the analysis of experimental magnetoconductance curves. We are able to find good agreement between theory and experiment, both yielding a fractal dimension $D \sim 1.3$.

\section{MODEL AND COMPUTATIONAL SCHEME}

We consider a model for a semiconductor stadium device fabricated in the 2DEG of a $\mathrm{AlGaAs} / \mathrm{GaAs}$ heterostructure similar to that in Ref. [10]. The Hamiltonian describing our 2D system reads in atomic units (a.u.)

$$
\hat{H}=\frac{1}{2}[-i \nabla+\mathbf{A}(\mathbf{r})]^{2}+V_{\text {ext }}(\mathbf{r}, t),
$$

where the vector potential is given in the linear gauge $\mathbf{A}(\mathbf{r})=$ $(-B y, 0,0)$ to describe a static and uniform magnetic field perpendicular to the plane. During the time propagation at $t>0$, the potential $V_{\text {ext }}(\mathbf{r}, t)$ consists of three parts: (i) a stadium with radius $r=1$ and width $d=0.7$, (ii) input and output leads of width $w=0.56$, and (iii) a linear potential along the propagation direction in the first two-thirds of the input lead describing a source-drain voltage. The used grid spacing in the system is $\Delta x=\Delta y=0.02$. The potential has hard boundaries with a depth $V_{0}=10000$ and the slope of the accelerating linear potential is -100 . The central part of the external potential is shown in Fig. 1. The input and output leads extend further to the left and right.

The initial state at $t=0$ is calculated by taking a small part of the input lead as a potential well. The resulting ground state of a single electron in the well is then used as an initial state for the time propagation. At $t>0$ the above described linear potential accelerates the wave packet across the system. For the time propagation we use a fourth-order Taylor expansion of
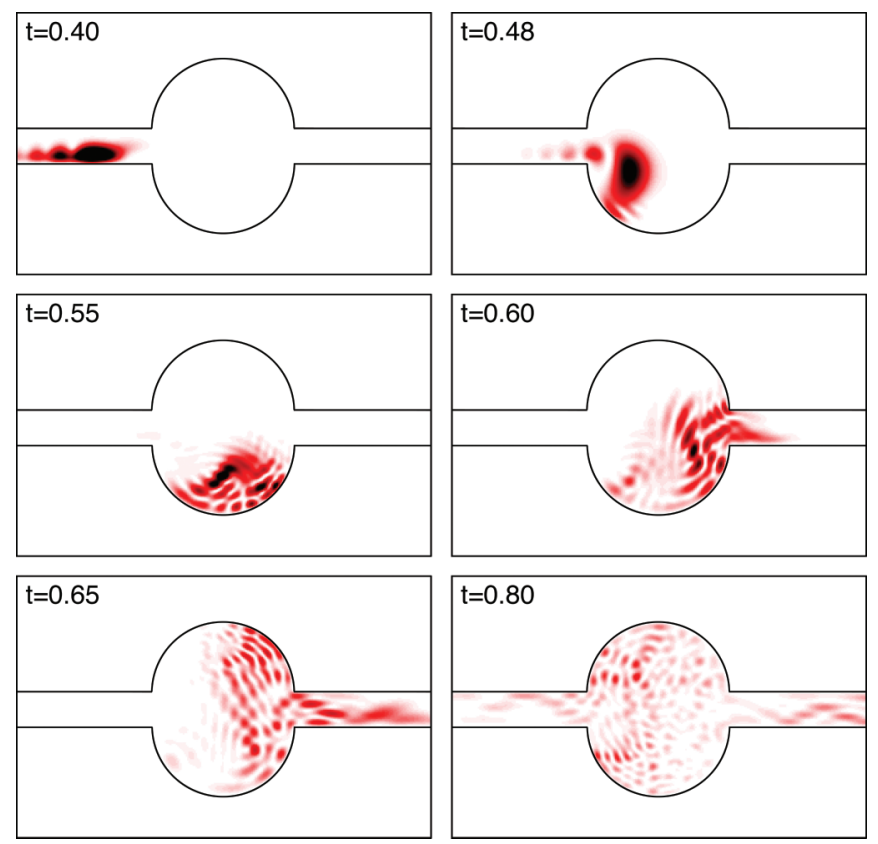

FIG. 1. (Color online) Snapshots of the electron density in the model stadium system (see the text) during a transport simulation with the magnetic flux $\Phi / \Phi_{0}=20$. The input and output leads extend further to the left and right. the time-evolution operator. The OCTOPUS code package [40] is used in all the calculations.

We assess the conductance by calculating the integrated probability density in the output lead from

$$
T(\Phi, t)=\int_{\text {output }} d \mathbf{r}|\psi(\Phi, \mathbf{r}, t)|^{2},
$$

where $\Phi$ is the fixed magnetic flux, given above in units of the magnetic flux quantum $\Phi_{0}=h / e$. We call $T$ a transmission factor assumed to be proportional to the transmission coefficient available in conventional transport theory. The validity of the transmission factor in estimating the relative conductivity as a function of an external parameter-here the magnetic flux-has been justified in Ref. [41]. Thus we repeat the time propagation for different values of $\Phi$ to obtain the magnetoconductance that can be compared with the experiments in Ref. [10].

It is important to note that our calculations allow energy dispersion for the wave functions in the cavity, i.e., we describe nonstationary states. In this respect, our results are not directly comparable to those of Ref. [22]. However, our transport approach qualitatively describes an experimental situation to the extent that each value for the magnetic field is treated equally, so that we can compare the relative conductance as a function of $B$. Previously, a similar approach was used to assess quantum conductance in quantum rings [41] and Aharonov-Bohm interferometers [42].

\section{TRANSPORT SIMULATIONS}

In Fig. 1 we show snapshots of the electron density at different times at $\Phi=20 \Phi_{0}$ through the stadium. Approximately one half of the density is transferred through and other half is either reflected back to the input lead or confined in the stadium. As expected, the density is scattered in the stadium in a chaotic fashion. The size of the wiggles during the scattering depends on the momentum: The higher the momentum the higher eigenstates are probed. We point out that the modes are not set prior to the calculation, but the wave packet is freely scattered and dispersed in the cavity. Here we have chosen the initial momentum of the wave packet such that considerable overlap is found with $\sim 50-100$ eigenstates of the stadium during the transport. This corresponds to considerable qualitative complexity in the propagated density, which, as shown below, leads to a complex behavior of $T$. We do not consider higher initial momenta in order to guarantee a reliable description of the scattered wave packet in the cavity with the used grid spacing.

A complete presentation of our transport results is given in Fig. 2, where the transmission factor $T$ is plotted as a function of both time and the magnetic flux. The figure consists of 401 respective time propagations, each with a fixed number of flux quanta $\Phi / \Phi_{0}$ ranging from zero to 40 in steps of 0.1 . The flux range is qualitatively similar to the experiment in Ref. [10]. A complex magnetoconductance is formed if the propagation time is larger than $\sim 1$. A cross section of the conductance at $t=1.4$ is shown in Fig. 3 . We point out that due to the finite system size we are not able to reach the equilibrium current and thus find the absolute conductance. In practice, we stop the time propagation immediately when the backscattering from 


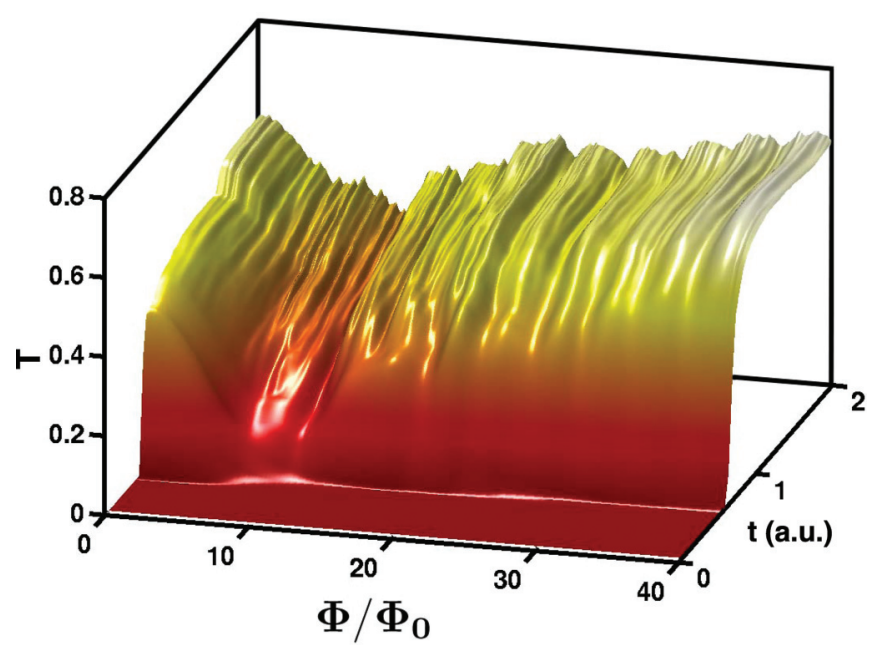

FIG. 2. (Color online) Transmission factor as a function of time and magnetic flux through the stadium.

the walls of the calculation box becomes visible. Therefore, we consider fixed propagation times through the parameter range of $\Phi / \Phi_{0}$. In other words, a fixed propagation time is expected to treat all the values of $\Phi / \Phi_{0}$ equally in order to obtain the relative conductance $T$.

We first briefly consider the general trends in $T$ in Fig. 3. As the flux is increased from zero the conductance decreases mainly due to the disappearance of trajectories directly coupling the left and the right leads. After reaching the minimum the conductance generally becomes larger, which is due to the increase of skipping orbits along the boundaries of the system. At large fields, interference effects play an important role [43]. We point out, however, that the dynamics is largely chaotic through the whole range of fluxes considered here, possibly only apart from the zero-flux limit.

Now the essential question is whether the conductance as a function of the magnetic flux shows fractal characteristics. Moreover, it is interesting to consider how large propagation times are required to find fractals. This is analyzed in the

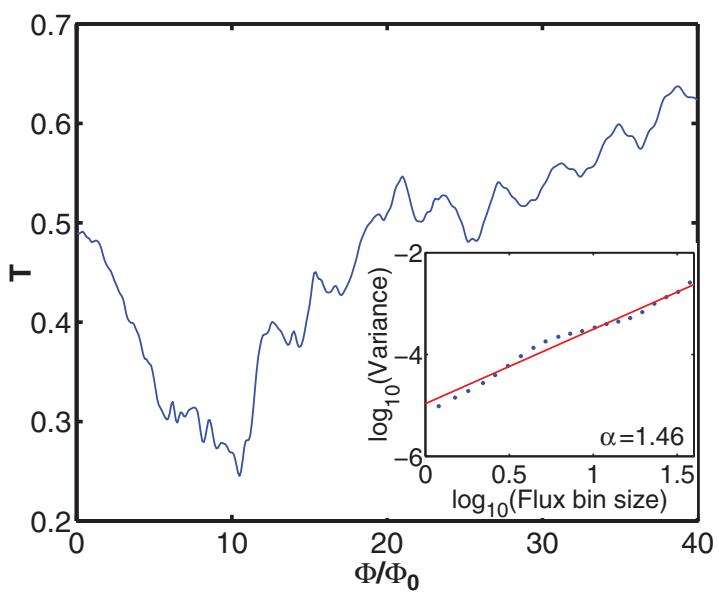

FIG. 3. (Color online) Transmission factor as a function of the magnetic flux at $t=1.4$. The inset shows the scaling exponent $\alpha=$ 1.46 obtained from the DFA analysis. following with two techniques: the variation method $[3,10,36]$ and DFA [37-39].

\section{METHODS FOR FRACTAL ANALYSIS}

\section{A. Variation method}

To extract the fractal dimension $D$ for a mapping $f$ : $\mathbb{R} \rightarrow \mathbb{R}$, the domain of the given function is first divided into length intervals $\Delta x$. The difference between the minimum and maximum of the function is calculated within every interval and added up. Note that the intervals are shifted windowwise across the $x$ axis (not pointwise). In the case of fractal scaling, the resulting sum is a power-law function of the interval length $[3,10,36]$ :

$$
\sum_{i}[\max f(x)-\min f(x)]_{\left|x-x_{i}\right|<\Delta x / 2} \propto(\Delta x)^{-D+1} .
$$

\section{B. Detrended fluctuation analysis}

Detrended fluctuation analysis is a standard method that was developed in the context of time-series analysis to study $1 / f$ noise and long-range correlations [37] and has proven to be very reliable particularly in dealing with nonstationary time series and trends in the data $[6,37,38,44,45]$. It has also been used outside the time domain, e.g., to study the organization of DNA nucleotides [39]. Here DFA is applied to fractal conductance curves.

The standard procedure of DFA consists of the following four steps [37,38]: (i) integrating the time series, (ii) dividing the series into windows of size $s$, (iii) fitting with a polynomial $f_{s}(i)$ of degree $m=2, \ldots, 4$ that represents the trend in the window, and (iv) calculating the variance with respect to the local trend $f_{s}(i)$ from

$$
\begin{aligned}
F(s) & =\left\langle\left[f(i)-f_{s}(i)\right]^{2}\right\rangle \\
& =\frac{1}{N-1} \sum_{i=1}^{N}\left[f(i)-f_{s}(i)\right]^{2} \propto s^{\alpha} .
\end{aligned}
$$

The key point in applying DFA to study conductance fluctuations is to relate the exponent $\alpha$ to the quantity of interest (here the fractal dimension $D)$. It is known that $D=2-\gamma / 2$ with $\left\langle(\Delta G)^{2}\right\rangle \propto(\Delta B)^{\gamma}[10,22]$. The latter is exactly step (iv) of the DFA analysis above. We therefore omit step (i) and identify $\alpha=\gamma$, hence the fractal dimension reads $D=2-\alpha / 2$.

\section{RESULTS ON THE FRACTALITY}

In DFA we apply quadratic detrending $(m=2)$ to our data in Fig. 3. The inset shows the fitting of the data (solid line) at $t=1.4$, which yields $\alpha=1.46$. This qualitatively agrees well with the experimental result $\gamma=\alpha=1.38$ of Sachrajda et al. [10]. The corresponding fractal dimension extracted from DFA is $D=1.27$. In comparison, the variation method yields $D=$ 1.32 for our data, whereas the corresponding experimental result, obtained with the same method, is $D=1.25$ [10]. The expected error bars for our results are discussed below. Nevertheless, we find excellent qualitative agreement of the results regarding both the different methods and comparison with the experimental data. We point out that our stadium 

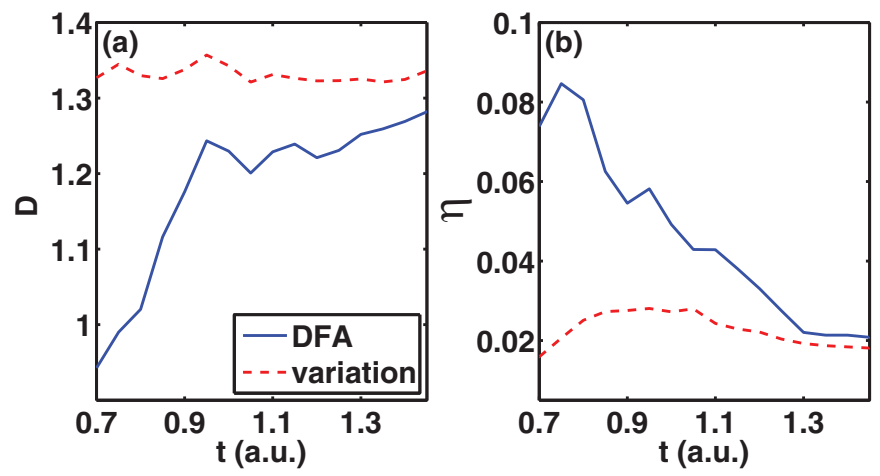

FIG. 4. (Color online) (a) Fractal dimension $D$ calculated from DFA with the relation $D=2-\alpha / 2$ and from the variation method, respectively, during the time propagation. Note that the fractal structure is developed only at $t \gtrsim 1$. (b) Time development of the error in the fitting procedure at $t=0.7, \ldots, 1.4$ (see the text).

model is similar to the experiment and the channel dimensions are also comparable. According to our calculations, increasing the channel width from 0.56 to 0.7 leads to the same $D$ obtained in the variation method, whereas DFA yields a slightly smaller $D$.

In Fig. 4(a) we show the time development of the fractal dimension obtained from DFA and the variation method, respectively. We point out that clear signatures of a fractal structure are developed only at $t \gtrsim 1$. Nevertheless, $D$ converges during the time propagation towards the values given above and the quality of the fitting in both methods improves as well. Figure 4(b) shows the error in the fitting $\eta=1-R^{2}$ for times $t=0.7, \ldots, 1.4$. Here $R$ is the Pearson product-moment correlation coefficient of the $\log$-log data. Thus $\eta$ measures the linear fit quality such that $\eta=0$ corresponds to exact linear behavior. The minimum of the error is obtained at $t \approx 1.4$, which is the optimal time used above to determine $\alpha$ and $D$. At larger times with $t \gtrsim 1.4$ the error increases due to backscattering effects resulting from the finite simulation box (see above). In this way we are able to determine the range of validity in our scheme to calculate the fractal dimension.

It is important to note that in addition to the numerical error of the fitting procedure (see above), the algorithms for fractal analysis have internal error bars analyzed in detail by Pilgram and Kaplan [46]. For example, DFA results for the fractal scaling are expected to have a standard deviation of $\sim 15 \%$ for data sets that are the of same size as ours. The results from the variational analysis are expected to contain similar deviations.

Finally we point out that qualitatively similar fractal dimensions have been obtained in various experiments on billiard systems of different shapes $[11,13,14]$. The dependence of $D$ on energy-level resolution determined by experimental conditions has been discussed in several works [47]. Moreover, the considerable role of disorder in the modulation-doped 2DEG was recently demonstrated [15]. However, in the same work it was shown that electrostatic doping leads to reproducible properties in thermal cycling. In view of these recent advances it can be expected that ballistic transport properties of 2DEG billiard systems will be determined in forthcoming experiments with a high precision. In turn, this motivates us to extend the applications of the present method to various geometries.

\section{SUMMARY}

We have calculated the time evolution of a single-electron wave packet through a two-dimensional stadium-shaped cavity by solving the Schrödinger equation in real time and real space. The relative conductance has been calculated for a large set of magnetic fluxes in order to analyze the fractal nature of the magnetoconductance. We have found that the conductance shows clear indications for fractal scaling. The fractal dimensions extracted from two respective methods are consistent with each other. Moreover, we have found excellent qualitative agreement with previous experimental results. Our findings indicate that DFA is well suited for the analysis of fractal scaling in chaotic quantum transport. Hence we suggest to extend the use of the concept of data detrending (and hence DFA) to study fractal scaling of transport and other characteristics in chaotic (quantum) systems.

\section{ACKNOWLEDGMENTS}

We thank Adam Micolich, Rainer Klages, and Andrew Sachrajda for very helpful comments and discussions. This work was supported by the Magnus Ehrnrooth Foundation, Wihuri Foundation, the Academy of Finland, and the European Community's FP7 through the CRONOS project, Grant Agreement No. 280879. H.H. acknowledges funding through the German Research Foundation (Grant No. 6312/1-2). CSC Scientific Computing Ltd. is acknowledged for computational resources.
[1] B. B. Mandelbrot, The Fractal Geometry of Nature (Freeman, New York, 1983).

[2] B. Mandelbrot, Science 156, 636 (1967).

[3] P. Meakin, Fractals, Scaling, and Growth Far from Equilibrium (Cambridge University Press, Cambridge, 1998).

[4] R. Voss and J. Clarke, Nature (London) 258, 317 (1975).

[5] K. J. Hsu and A. J. Hsu, Proc. Natl. Acad. Sci. USA 87, 938 (1990).

[6] H. Hennig, R. Fleischmann, A. Fradebohm, Y. Hagmayer, J. Nagler, A. Witt, F. J. Theis, and T. Geisel, PLoS ONE 6, e26457 (2011)
[7] H. Hennig, R. Fleischmann, and T. Geisel, Phys. Today 65(7), 64 (2012).

[8] T. Michely and J. Krug, Islands, Mounds, and Atoms: Patterns and Processes in Crystal Growth Far from Equilibrium (Springer, Berlin, 2004).

[9] M. F. Barnsley, Fractals Everywhere, 2nd ed. (Academic Press, Boston, 1993).

[10] A. S. Sachrajda, R. Ketzmerick, C. Gould, Y. Feng, P. J. Kelly, A. Delage, and Z. Wasilewski, Phys. Rev. Lett. 80, 1948 (1998).

[11] A. P. Micolich, R. P. Taylor, R. Newbury, J. P. Bird, R. Wirtz, C. P. Dettmann, Y. Aoyagi, and 
T. Sugano, J. Phys.: Condens. Matter 10, 1339 (1998).

[12] H. Hegger, B. Huckestein, K. Hecker, M. Janssen, A. Freimuth, G. Reckziegel, and R. Tuzinski, Phys. Rev. Lett. 77, 3885 (1996).

[13] A. P. Micolich, R. P. Taylor, T. P. Martin, R. Newbury, T. M. Fromhold, A. G. Davies, H. Linke, W. R. Tribe, L. D. Macks, C. G. Smith, E. H. Linfield, and D. A. Ritchie, Phys. Rev. B 70, 085302 (2004).

[14] C. A. Marlow, R. P. Taylor, T. P. Martin, B. C. Scannell, H. Linke, M. S. Fairbanks, G. D. R. Hall, I. Shorubalko, L. Samuelson, T. M. Fromhold, C. V. Brown, B. Hackens, S. Faniel, C. Gustin, V. Bayot, X. Wallart, S. Bollaert, and A. Cappy, Phys. Rev. B 73, 195318 (2006).

[15] A. M. See, I. Pilgrim, B. C. Scannell, R. D. Montgomery, O. Klochan, A. M. Burke, M. Aagesen, P. E. Lindelof, I. Farrer, D. A. Ritchie, R. P. Taylor, A. R. Hamilton, and A. P. Micolich, Phys. Rev. Lett. 108, 196807 (2012).

[16] M. V. Berry, J. Phys. A 29, 6617 (1996).

[17] D. Wójcik, I. Białynicki-Birula, and K. Życzkowski, Phys. Rev. Lett. 85, 5022 (2000).

[18] L. Hufnagel, R. Ketzmerick, and M. Weiss, Europhys. Lett. 54 703 (2001).

[19] G. Benenti, G. Casati, I. Guarneri, and M. Terraneo, Phys. Rev. Lett. 87, 014101 (2001).

[20] I. Guarneri and M. Terraneo, Phys. Rev. E 65, 015203 (2001).

[21] E. Louis and J. A. Vergés, Phys. Rev. B 61, 13014 (2000).

[22] R. Ketzmerick, Phys. Rev. B 54, 10841 (1996).

[23] H. Hennig, R. Fleischmann, L. Hufnagel, and T. Geisel, Phys. Rev. E 76, 015202(R) (2007).

[24] A. Bäcker, A. Manze, B. Huckestein, and R. Ketzmerick, Phys. Rev. E 66, 016211 (2002).

[25] C. M. Marcus, A. J. Rimberg, R. M. Westervelt, P. F. Hopkins, and A. C. Gossard, Phys. Rev. Lett. 69, 506 (1992).

[26] A. P. Micolich, R. P. Taylor, A. G. Davies, J. P. Bird, R. Newbury, T. M. Fromhold, A. Ehlert, H. Linke, L. D. Macks, W. R. Tribe, E. H. Linfield, D. A. Ritchie, J. Cooper, Y. Aoyagi, and P. B. Wilkinson, Phys. Rev. Lett. 87, 036802 (2001).

[27] Y. Takagaki, M. ElHassan, A. Shailos, C. Prasad, J. P. Bird, D. K. Ferry, K. H. Ploog, L.-H. Lin, N. Aoki, and Y. Ochiai, Phys. Rev. B 62, 10255 (2000).

[28] U. Kuhl, H.-J. Stöckmann, and R. Weaver, J. Phys. A 38, 10433 (2005).
[29] H. U. Baranger and P. A. Mello, Phys. Rev. Lett. 73, 142 (1994).

[30] S. Heusler, S. Müller, P. Braun, and F. Haake, Phys. Rev. Lett. 96, 066804 (2006)

[31] G. Casati, I. Guarneri, and G. Maspero, Phys. Rev. Lett. 84, 63 (2000).

[32] Ph. Jacquod and R. S. Whitney, Phys. Rev. B 73, 195115 (2006).

[33] P. W. Brouwer and S. Rahav, Phys. Rev. B 74, 075322 (2006).

[34] For recent reviews on quantum transport, see, e.g., M. Di Ventra, Electrical Transport in Nanoscale Systems (Cambridge University Press, Cambridge, 2008); G. Stefanucci and R. van Leeuwen, Nonequilibrium Many-Body Theory of Quantum Systems: A Modern Introduction (Cambridge University Press, Cambridge, 2013).

[35] P. J. J. Luukko and E. Räsänen, Comput. Phys. Commun. 184, 769 (2013).

[36] B. Dubuc, J. F. Quiniou, C. Roques-Carmes, C. Tricot, and S. W. Zucker, Phys. Rev. A 39, 1500 (1989).

[37] C. K. Peng, S. Havlin, H. E. Stanley, and A. L. Goldberger, Chaos 5, 82 (1995).

[38] J. W. Kantelhardt, E. Koscielny-Bunde, H. H. A. Rego, S. Havlin, and A. Bunde, Physica A 295, 441 (2001).

[39] C.-K. Peng, S. V. Buldyrev, S. Havlin, M. Simons, H. E. Stanley, and A. L. Goldberger, Phys. Rev. E 49, 1685 (1994).

[40] M. A. L. Marques, A. Castro, G. F. Bertsch, A. Rubio, Comput. Phys. Commun. 151, 60 (2003); A. Castro, H. Appel, M. Oliveira, C. A. Rozzi, X. Andrade, F. Lorenzen, M. A. L. Marques, E. K. U. Gross, and A. Rubio, Phys. Status Solidi B 243, 2465 (2006).

[41] V. Kotimäki and E. Räsänen, Phys. Rev. B 81, 245316 (2010).

[42] V. Kotimäki, E. Cicek, A. Siddiki, and E. Räsänen, New J. Phys. 14, 053024 (2012).

[43] H. van Houten, C. W. J. Beenakker, J. G. Williamson, M. E. I. Broekaart, P. H. M. van Loosdrecht, B. J. van Wees, J. E. Mooij, C. T. Foxon, and J. J. Harris, Phys. Rev. B 39, 8556 (1989).

[44] H. D. Jennings, P. C. Ivanov, A. M. Martins, P. C. Silva, and G. M. Viswanathan, Physica A 336, 585 (2004).

[45] P. Ch. Ivanov, Q. D. Y. Ma, R. P. Bartsch, J. M. Hausdorff, L. A. Nunes Amaral, V. Schulte-Frohlinde, H. E. Stanley, and M. Yoneyama, Phys. Rev. E 79, 041920 (2009).

[46] B. Pilgram and D. T. Kaplan, Physica D 114, 108 (1997).

[47] For a review, see A. P. Micolich, A. M. See, B. C. Scannell, C. A. Marlow, T. P. Martin, I. Pilgrim, A. R. Hamilton, H. Linke, and R. P. Taylor, Fortschr. Phys. 61, 332 (2013). 